\title{
TRANSIENT 3-D THERMAL ANALYSIS FOR AN AIR-COOLED INDUCTION MOTOR
}

\author{
By: \\ H-P. Liu \\ V. Lelos \\ C.S. Hearn
}

IEEE International Electric Machines and Drives Conference, May 15-18, 2005, San Antonio, Texas, U.S.A.

PR -381

Center for Electromechanics

The University of Texas at Austin

PRC, Mail Code R7000

Austin, TX 78712

(512) 471-4496 


\title{
Transient 3-D Thermal Analysis for an Air-Cooled Induction Motor
}

\author{
H-P. Liu, V. Lelos, and C.S. Hearn \\ Center for Electromechanics, The University of Texas at Austin \\ 1 University Station, R7000 \\ Austin, TX 78712
}

\begin{abstract}
For an induction motor that is cooled by an airflow ventilated across the air gap between the rotor and stator, the rotor endring and stator end-turn cooling might influence the motor temperatures in the core sections appreciably. Detailed motor thermal modeling is typically required to provide transient rotor temperature distributions for rotor structural analysis and to predict stator temperature distributions to assure the maximum stator temperature is below the stator insulation temperature limit.

For an air-cooled induction motor, transient 3-D finite element thermal analyses for both rotor and stator have been performed in this paper. In the stator thermal model, the slot conductors and various insulations were smeared and equivalent thermal properties were used to reduce modeling and computational effort. Position-dependent and time-dependent air cooling boundary conditions, which include air temperatures and forced convection heat-transfer coefficients, were applied to the 3-D thermal models.
\end{abstract}

\section{INTRODUCTION}

An induction motor has been designed to be an integral part of a large energy storage generator. Active air coolant, pressurized and circulated by an external fan, is introduced from one end of the motor. The air is directed to impinge on the stator end turns and rotor end ring at the air inlet, ventilate through the rotor/stator air gap, and flow across the end turns and end ring at the other end of the motor. The rotor and stator losses are removed by forced air convection. Airflow network modeling of the entire energy storage system was carried out to determine the induction motor air-gap air flow rate.

The induction motor was designed to provide torque to overcome windage and bearing losses at the peak rotor speed under "No Load" condition. As a transient load is applied, the generator is decelerated to a lower speed, and more torque is required to be provided by the induction motor to accelerate the generator back to the peak speed within a certain time period. This transient load occurs under a certain repetition rate for many-hour operations. The induction motor needs to be designed to supply time-dependent torques under "No Load" and "Load" conditions.

Other than the windage loss, the heat losses generated in various motor components, which include rotor bars and end rings, rotor lamination, stator winding, and stator lamination, need to be continuously removed by the forced airflow. The motor electrical design can initially be guided by transient 2D planar thermal analyses, in which the stator slot conductors

\footnotetext{
This research is sponsored by the U.S. Navy through General Atomics under contract number N68335-04-C-0167.
}

and insulations can be modeled in a detailed manner. However, the 2-D planar thermal results are not capable of providing critical thermal information in the rotor end-ring and stator end-turn regions. A 3-D thermal analysis becomes especially important when the axial heat conduction in the induction motor is considered to be nonnegligible due to significant variations of air cooling boundary conditions along the airflow paths across the induction motor.

\section{INDUCTION MOTOR DESIGN AND LOSSES}

The rotor core is made of M-19 laminate. There are a total of 58 rectangular C10200 oxygen-free copper bars in the rotor. Rotor end rings, made of this copper, are supported by alloy steel banding. There is no thermal insulation in the rotor conductor slots, except for certain thermal resistances caused by the assembly tolerances between the rotor copper bars and rotor lamination. The copper conductors are extended to a rotor outer surface area and part of the conductor surfaces are in direct contact with the air coolant in the air gap.

The stator winding is made of rectangular copper conductors. There are twelve copper conductors $2.59 \mathrm{~mm} \mathrm{x}$ $7.54 \mathrm{~mm}$ (0.102 in. $\times 0.297$ in., bare) in each half of the slot. Each conductor has a $0.076 \mathrm{~mm}$ (0.003 in.) Kapton conductor insulation wrap and a $0.102 \mathrm{~mm}$ (0.004 in.) NOMEX 410 turn wrap. These twelve conductors form a coil, which has a $0.396 \mathrm{~mm}$ (0.0156 in.) MICA tape coil wrap. The coil also has a $0.254 \mathrm{~mm}$ (0.010 in.) fiberglass tape armor coil wrap. In each stator slot, two coils are separated by a mid stick. The stator is vacuum impregnated and the dimensional and assembly tolerances are filled with a potting resin. The overall stator copper conductor packing factor in the stator slots is $66 \%$. The induction motor stator slot details are shown in Fig. 1.

Frictional windage losses result from a steady and relative motion of two surfaces that are separated by a fluid. In induction motor operations, windage losses are generated at those places where relative motions exist between the solid surfaces and the surrounding air. A significant portion of the total windage loss occurs in the annular air gap between the rotor and stator due to the high rotor surface velocity and relatively small air-gap size. The annular air gap flow between concentric cylinders with a rotating inner cylinder and a stationary outer cylinder is commonly 
referred as a Couette flow. For a continuum flow between concentric rotating cylinders, secondary flow of rows of circumferential Taylor vortices in the air gap due to centrifugal flow instability of a curved flow at relatively high rotating speeds will typically be formed.

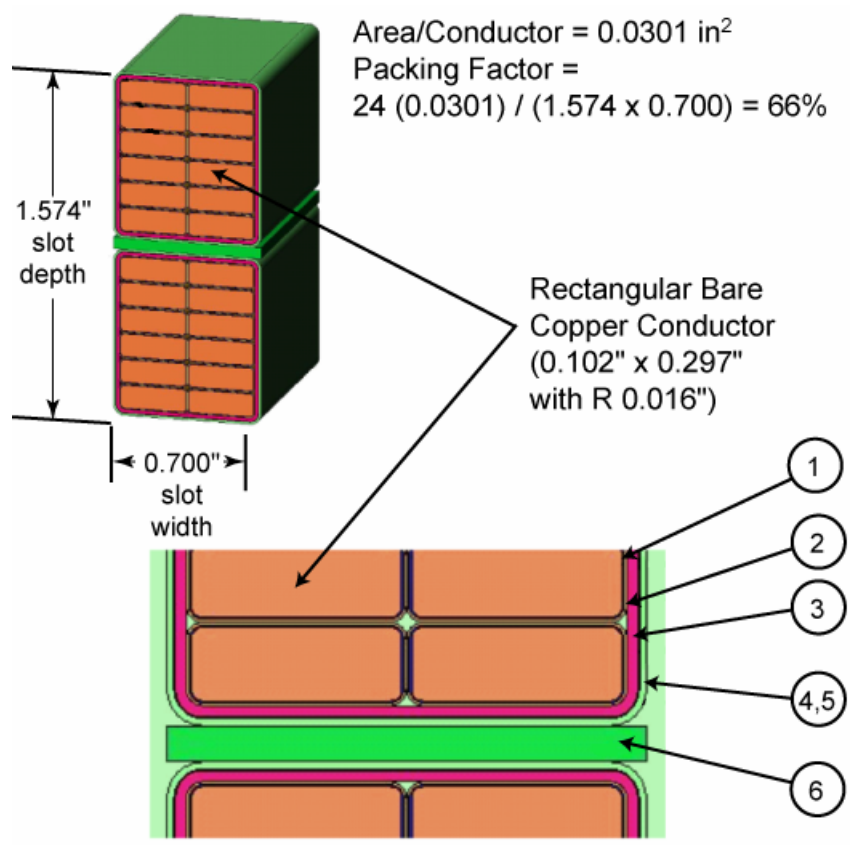

1. Conductor/Turn wrap - Austral Von Roll//sola 150FCR Kapton, provided on conductor (single 50\% lap) - $0.003 "$

2. Conductor/Turn Wrap - Nomex 410 (single 1/2 lap) - 0.004"

3. Coil Wrap - Von Roll/Isola mica mat 77642 tape (66\% lap wrap) -0.0156

4. Coil Head Wrap - 100CR Kapton, single half-lap, extending from coil end back to iron $-0.002 "$

5. Armor Coil Wrap - Medium weave glass tape (single 1/2 lap) $-0.010^{\prime \prime}$

6. Slot Mid Stick - Port City Grade PCGP-HT (232C) - 0.050" x 0.700

7. Vacuum impregnate entire motor stator with Von Roll Isola VRI 777

solventless polyester resin. Stator rotated during cure.

Fig. 1. Induction motor stator slot details.

For an annular rotating continuum flow, various flow regimes exist, such as laminar, transition, vortex, and turbulent flows. A laminar flow between rotating cylinders breaks down at certain rotational speeds and a stable flow of rows of circumferential Taylor vortices is formed. Couettetype turbulent flow only occurs at very high rotational speeds. For non-ventilated enclosed disks, the presence of four different flow regimes, laminar flow merged boundary layers, laminar flow separated boundary layers, turbulent flow merged boundary layers, and turbulent flow separated boundary layers, have been experimentally verified, and empirical disk torque coefficients in these flow regimes have been obtained. Calculations of continuum flow windage drags on smooth rotating cylinders and disks can be carried out by using empirical torque coefficients [1].

The University of Texas Center for Electromechanics (UTCEM) has designed and conducted a series of composite rotor spin tests to measure the windage losses and temperature distributions of a test setup at high rotor speeds and low air pressures. An analytical approach has been developed to predict high speed windage losses (rotor tip velocities up to $900 \mathrm{~m} / \mathrm{s}$ ) at low rotor cavity air pressures (0.1 torr to 10 torr) with windage tests conducted in both the continuum-flow and slip-flow regimes [2].

Smooth rotating surfaces introduce minimum windage friction resistances and can serve as a datum for judging the surface roughness effect on the windage losses. To consider the effect of surface roughness caused by the stacking of rotor and stator laminates on the windage drags, experimental data on a relationship between torque coefficient and Taylor number for rotating concentric cylinders which are physically rough [3] have been utilized to determine the induction motor windage loss.

The induction motor component heat losses, calculated during a five-hour operation at elevated temperatures, are included in Table I. The total motor losses generated in the two-hour (no load) and the remaining three-hour (under load) periods are $15.5 \mathrm{~kW}$ and $24.4 \mathrm{~kW}$, respectively. The rotor lamination losses are uniformly distributed in the rotor laminate teeth region. The stator lamination losses are nonuniformly divided between the teeth and yoke regions based on electromagnetic analysis results.

\section{TRANSIENT 3-D Thermal MODELING}

Since the stator end-turn and rotor end-ring cooling could influence the motor temperatures in the core sections, 3-D finite element thermal modeling for both the induction motor rotor and stator have been developed, and transient 3$\mathrm{D}$ thermal analyses have been performed. The rotor components are relatively simple and have been modeled individually in the rotor thermal analysis. An air layer $0.0635 \mathrm{~mm}$ (0.0025 in.) thick was assigned in between the rotor bars and rotor lamination, and was also allocated in between the rotor bars and steel bandings. It is not practical to consider the stator slot conductor and insulation details in a transient 3-D stator thermal model; instead, the slot conductors and various insulations were smeared and equivalent thermal properties were used to reduce modeling and computational effort. Due to geometrical symmetries, only sectors of the rotor and stator were actually modeled. Position-dependent and time-dependent air cooling boundary conditions, which include air temperatures and forced convection heat-transfer coefficients, were applied to the transient 3-D rotor and stator thermal models.

TABLE I

INDUCTION MOTOR HEAT LOSSES

\begin{tabular}{|l|r|r|}
\hline \multicolumn{1}{|c|}{ Loss component } & $\begin{array}{c}0 \text { to } 2 \mathrm{hr} \\
\text { no load @ } \\
\text { peak speed } \\
\text { (W) }\end{array}$ & $\begin{array}{c}2 \text { to } 5 \mathrm{hr} \\
\text { under } \\
\text { load } \\
\text { (W) }\end{array}$ \\
\hline Rotor bars and end rings & 639 & 2,804 \\
\hline Rotor lam. teeth & 2,900 & 4,423 \\
\hline Stator winding & 3,301 & 7,372 \\
\hline Stator lam. teeth and yoke & 7,132 & 8,239 \\
\hline Windage & 1,567 & 1,567 \\
\hline Total loss & 15,539 & 24,405 \\
\hline
\end{tabular}

A system-level flow network modeling software [4] has been used to perform airflow analysis in the large energy 
storage generator. The airflow system was graphically represented as a network of components and flow paths that were selected from an included library. The characteristics of the network components and flow paths were defined according to the generator design. The airflow system was converted into a network of nodes and links. Conservations of mass, momentum, and energy were enforced over these nodes and links. An induction motor air flow rate of $29 \mathrm{~m}^{3} / \mathrm{min}$ (1,030 standard $\left.\mathrm{ft}^{3} / \mathrm{min}\right)$ was predicted from this airflow network analysis and was used in transient energy balance calculations to determine the position and time dependent air temperatures. The forced convection heat-transfer coefficients are air temperature dependent and rotating configuration dependent, such as rotating cylinders or rotating disks in enclosures [5, 6]. The air cooling boundary conditions for the transient 3-D rotor and stator thermal analyses are shown in Figs. 2 and 3. Referring to Figs. 2 and 3 , the motor end which has low air temperatures is the end for the air inlet, and high exhausted air temperatures are observed at the other end.

\section{Thermal Analysis Results}

After applying no-load rotor and stator losses at the rotor peak speed for two hours, both the rotor and stator essentially reach steady-state temperature distributions. During the entire five-hour transient thermal analysis period, maximum rotor temperature of $108^{\circ} \mathrm{C}$ and stator temperature of $173^{\circ} \mathrm{C}$ occur at the time of 4.8 hours. The rotor temperature distribution at 4.8 hours after the initial operation is plotted in Fig. 4. Transient maximum rotor-bar and end-ring temperatures are plotted in Fig. 5. The stator temperature distribution at 4.8 hours after the initial operation is plotted in Fig. 6. Transient maximum stator lamination, stator coil insulation, and mid stick temperatures are plotted in Fig. 7. The temperature dips in Figs. 5 and 7 are caused by momentary no-load operations.

\section{CONCLUSIONS}

Transient 3-D thermal analyses of an induction motor were performed to investigate rotor end-ring cooling, stator endturn cooling, and axial conduction cooling effect on the rotor and stator temperatures in the core region. Both position and time dependent thermal loads and air cooling boundary conditions are considered in the thermal modeling. Rotor temperatures are acceptable as results of relatively low thermal loads and lack of thermal insulation. High stator temperatures occur in the lamination region, other than the slot conductors in the core region, is primarily caused by the high lamination losses, poor transverse thermal conductivity of the stator laminate, and end-turn cooling effect on the stator conductors in the core region.

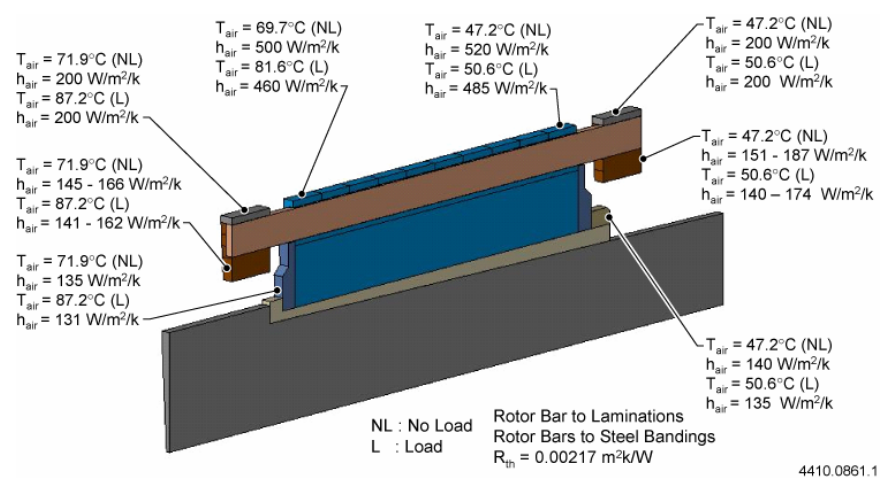

Fig. 2. Air cooling boundary condition for transient rotor thermal analysis.

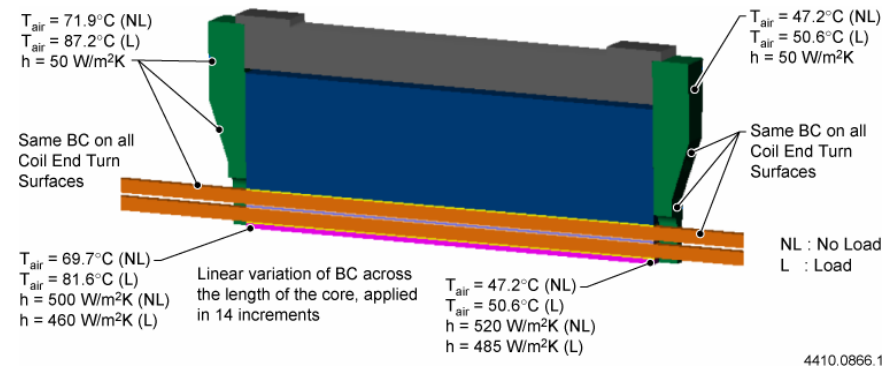

Fig. 3. Air cooling boundary condition for transient stator thermal analysis.

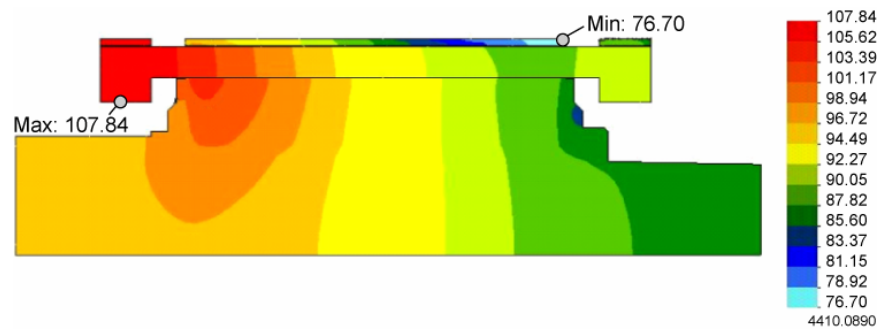

Fig. 4. Induction motor rotor temperature $\left({ }^{\circ} \mathrm{C}\right)$ distribution (time $=4.8 \mathrm{hr}$ ).

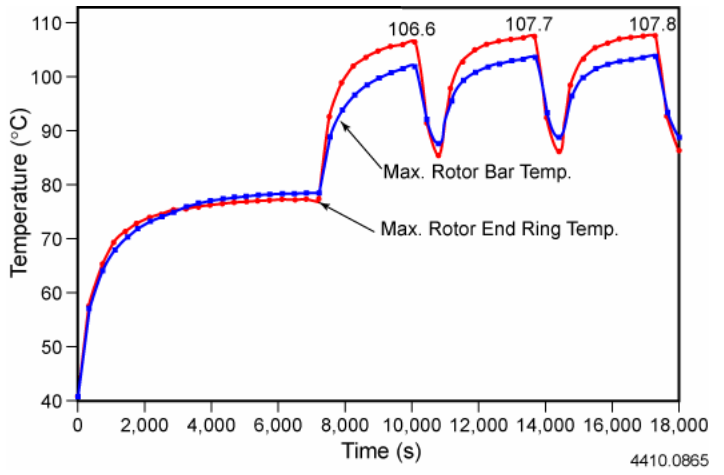

Fig. 5. Induction motor rotor transient temperatures. 


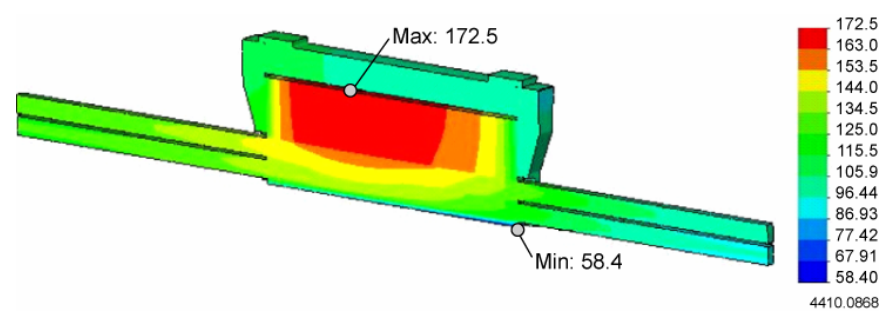

Fig. 6. Induction motor stator temperature $\left({ }^{\circ} \mathrm{C}\right)$ distribution (time $=4.8 \mathrm{hr}$ ).

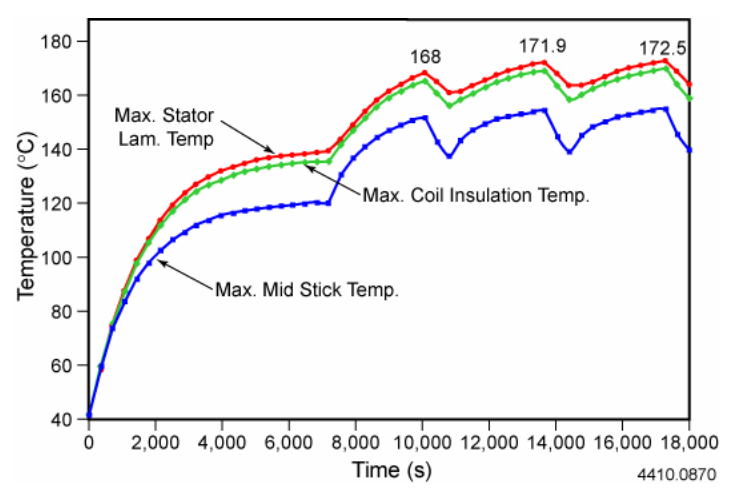

Fig. 7. Induction motor stator transient temperatures.

\section{REFERENCES}

[1] The General Electric Fluid Flow Data Book, Genium Publishing Corporation, Schenectady, New York, 1994.

[2] H-P Liu, M. D. Werst, J. J. Hahne, and D. Bogard, "Prediction of windage losses of an enclosed high speed composite rotor in low air pressure environments," ASME Summer Heat Transfer Conference, Las Vegas, NE, July 21-23, 2003.

[3] D. G. McLaren and H. J. Leutheusser, "Hydrogenerator windage loss," General Papers in Fluids Engineering, ASME FED-Vol. 127, 1991.

[4] MacroFlow - A System Modeling and Analysis Tool, Innovative Research, Inc.

[5] C. Gazley, Jr., "Heat-transfer characteristics of the rotational and axial flow between concentric cylinders,” ASME Transactions, vol. 80, No. 1, pp. 7990, January 1958.

[6] C. L. Ong, and J. M. Owen, "Computation of the flow and heat transfer due to a rotating disc," International Journal of Heat and Fluid Flow, vol. 12, No. 2, pp. 106-115, June 1991. 